\title{
Investigation of the Protective Effects of Caffeic Acid Phenethyl Ester against Cisplatin-induced Liver Damage in Rats
}

\author{
Tayfun Ceylan ${ }^{\mathbf{1} 2^{*}}$, Birkan Yakan²
}

${ }^{1}$ Cappadocia University, Cappadocia Vocational School, Department of Medical Services and Technique, Pathology Laboratory Techniques Pr., Nevşehir, Turkey.

${ }^{2}$ Erciyes University, Faculty of Medicine, Department of Histology \& Embryology, Kayseri, Turkey.

\begin{abstract}
Cisplatin (CP) is used as an effective chemotherapeutic drug in the treatment of various solid tumors. However, side effects such as hepatotoxicity limit the use of the drug. We investigated the protective effects of caffeic acid phenethyl ester (CAPE), one of the active ingredients of propolis, against hepatotoxicity caused by CP treatment in the liver. 38 Wistar albino rats were divided into 4 groups. Control group was given physiological saline solution for 12 day. CP group was given a single dose of CP $(7 \mathrm{mg} / \mathrm{kg})$ on the day 7. CP+CAPE group, was given CAPE (10 $\mu \mathrm{mol} / \mathrm{kg} / \mathrm{day})$ for 12 days and a single dose of CP (7 mg/kg) on day 7. CAPE group received CAPE (10 $\mu \mathrm{mol} / \mathrm{kg} /$ day) for 12 days. Livers of rats sacrificed on the 14th day were stained with hematoxylin-eosin after histological tissue follow-up. The preparations were evaluated and scored. Rat weights were measured and recorded at the beginning and end of the experiment. CP caused significant histopathological changes in the liver. CP also prevented the increase in rat weight. CAPE played an effective role as a protective agent against the histopathological changes caused by CP and showed signs of tissue healing. Our results show that CAPE can be protective against hepatotoxicity associated with CP.
\end{abstract}

Key words: Caffeic acid phenethyl ester, Cisplatin, Hepatotoxicity, Liver damage, Rat.

\section{Introduction}

Cisplatin (CP) is an important antineoplastic drug used in the treatment of solid tumors (1). The drug, which was first successfully used in cancer patients in 1971, was approved by the American Food and Drug Administration (FDA) in 1978 (2). CP or cis-diamminedichloroplatinum (II) is one of the effective chemotherapeutic drugs widely used in the treatment of testicular, bladder, head, neck, lung, breast and ovarian cancers. However, its negative side effects such as nephrotoxicity, ototoxicity and cardiomyopathy limit its clinical use. (3, 4). It is widely known that $\mathrm{CP}$ is significantly retained in the human liver and high doses of the drug cause

\footnotetext{
* Corresponding author: Tayfun Ceylan, Cappadocia University, Cappadocia Vocational School, Department of Medical Services and Technique, Pathology Laboratory Techniques Pr., Nevşehir, Turkey. Email: tyf.ceylan@gmail.com, ORCID ID: 0000-0002-0917-0378.
} 
hepatotoxicity. (5). Although dose-related side effects and ototoxicity are important limiting factors in cancer treatment, recent studies have confirmed that hepatotoxicity is another important dose-limiting side effect of CP-based chemotherapy. However, there is a little research on CPinduced hepatotoxicity and the underlying mechanism remains unclear (6).

Caffeic acid phenethyl ester (CAPE) is one of the active ingredients of the pungent and fragrant propolis substance found in the extract collected by bees from plants. CAPE has been used as a folk remedy for many years (7). CAPE has many biological activities such as anticancer, antioxidant, anti-inflammatory, immune stimulator, and antifungal, antiviral and antibacterial. The mechanism of biological effects of CAPE is not fully known $(8,9)$. CAPE is known to be cytotoxic to many types of cancer cells, including breast cancer cells, but it has not been shown to have any toxic effects on healthy tissues (9).

In this study, we evaluated protective effects of CAPE against hepatotoxicity caused by $\mathrm{CP}$, which is used as an anticarcinogenic drug in the treatment of many cancer types.

\section{Material and methods}

\section{Animals and drug administration}

The study protocol was accepted by the Experimental Animal and Local Ethics Committee of Erciyes University (decision no: 15/59). Herein, 38 male adult Wistar albino rats, weighing between 150 and 220 $\mathrm{g}$, and aged between 8 and 10 weeks, were supplied by the Experimental Animal Laboratory of Erciyes University. The rats were housed at $20-22^{\circ} \mathrm{C}$ under a $12: 12$ light/dark photoperiod and fed ad libitum. The animals were randomly divided into 4 groups. Body weights of animals in each group were measured and recorded. Control group $(\mathrm{n}=8)$ was given a physiological saline solution $(1 \mathrm{ml} / \mathrm{kg} /$ day $)$ intraperitoneally (i.p.) for 12 day. CP group $(n=10)$ was given a single dose of CP (Koçak Pharma, İstanbul, Turkey) (7 $\mathrm{mg} / \mathrm{kg}$ ) i.p. on day 7 of the experiment. The dose of $\mathrm{CP}$ was selected according to a previous study that demonstrated significant hepatotoxicity in rats (3). $\mathrm{CP}+\mathrm{CAPE}$ group $(\mathrm{n}=10)$ was given $\mathrm{CAPE}$ (Sigma-Aldrich, St. Louis, MO, USA) (10 $\mu \mathrm{mol} / \mathrm{kg} /$ day) i.p. for 12 days and single dose of CP $(7 \mathrm{mg} / \mathrm{kg})$ i.p. on day 7 of the experiment. The dose of CAPE was chosen according to the results of the studies in which this agent has a corrective effect (10). CAPE group $(n=10)$ was given CAPE (10 $\mu \mathrm{mol} / \mathrm{kg} /$ day) i.p. for 12 days. Before animals were sacrificed, the body weights were measured and recorded. At the end of the study (14th day), rats were decapitated after intraperitoneal ketamine $(75 \mathrm{mg} / \mathrm{kg})$ xylazine $(10 \mathrm{mg} / \mathrm{kg})$ anesthesia, and the liver tissues were rapidly removed. The sections obtained from the livers were stained with hematoxylin-eosin (HE) and histological damage was evaluated.

Hematoxylin-eosin staining

First, the $5 \mu \mathrm{m}$ sections taken from the paraffin blocks were spread out on slides. Standard histological methods were applied to the prepared slides. The paraffin was removed with xylol and passed through a graded alcohol series and diluted. The sections were stained with $H \& E$ to observe the general histological structure. The sections were examined after passing through an increasing alcohol series and xylene. H\&E staining was purchased from Nanotek Lab (Kayseri, Turkey) (11). Then, images of the slides were taken under the Olympus BX51 light microscope (Tokyo, Japan) with a DP71 
model digital camera.

The liver tissue structure was examined and randomly evaluated with standard light microscopy and it was scored by the study group. While applying histopathological score, the following criteria were used; hemorrhage, necrotic hepatocytes, vacuolized hepatocytes, and the appearance of hepatocyte cords. Scoring was conducted as follows: 0: not at all, 1: $0-25 \%, 2: 26-50 \%, 3: 51-75 \%$, and $4: 76-$ $100 \%$ (12).

\section{Statistical analysis}

All statistical analyses were carried out using SPSS statistical software (SPSS for Windows, SPSS Inc, Chicago, IL, version 21.0). The Kolmogorov-Smirnov and Shapiro-Wilk tests were used to identify normal distribution of the data. In case of normal distribution, quantitative variables were compared using One-way analysis of variance (ANOVA) and post hoc Tukey test. Descriptive statistics were shown as mean \pm standard deviation (SD). Statistical significance was defined as $p<0.05$.

\section{Results}

Light microscopic examination
Light microscopic evaluation was made on preparations stained with H\&E. The preparations were evaluated at $\times 200$ and $\times 400$ magnifications. The tissue sections in the Control and CAPE groups have normal histological appearance. Hemorrhage, sinusoidal dilatation and irregular sinusoids were observed in the tissue sections belonging to the $\mathrm{CP}$ group. In addition, eosinophilic necrotic hepatocytes and vacuolization were also observed in the CP group. Hemorrhage, sinusoidal dilatation, eosinophilic hepatocytes and vacuolization were reduced in the tissue sections belonging to the $\mathrm{CP}+\mathrm{CAPE}$ group when compared with the $\mathrm{CP}$ group. $\mathrm{H} \& \mathrm{E}$ staining of the liver tissues in the experimental groups are shown in the Figure 2 and Figure 3. In addition, liver histopathological scoring was significantly higher in the CP group compared to the Control group $(p<0.001)$. On the contrary, it was significantly lower in the CP+CAPE group when compared with the $\mathrm{CP}$ group $(p<0.001)$. Liver histopathological scoring did not show a significant difference among the Control, CAPE and $\mathrm{CP}+\mathrm{CAPE}$ groups. The data of liver histopathological scoring are shown in Table 1 and Figure 1.

Table 1: Initial and final body weights and histopathological score among experimental groups.

\begin{tabular}{|c|c|c|c|c|}
\hline & Initial Weight (g) & Final Weight (g) & Histopathological score & p value \\
\hline Control & $242.87 \pm 15.35$ & $273.87 \pm 14.78^{*}$ & $0.52 \pm 0.65^{\mathrm{a}}$ & $<0.001$ \\
\hline $\mathbf{C P}$ & $219.75 \pm 14.92$ & $234.62 \pm 18.32$ & $2.27 \pm 1.00^{\mathrm{b}}$ & $<0.001$ \\
\hline $\mathrm{CP}+\mathrm{CAPE}$ & $209.83 \pm 17.31$ & $218.83 \pm 15.21$ & $0.96 \pm 0.90^{\mathrm{a}}$ & $<0.001$ \\
\hline CAPE & $210.25 \pm 10.15$ & $234.62 \pm 9.89 *$ & $0.78 \pm 0.78^{a}$ & $<0.001$ \\
\hline
\end{tabular}




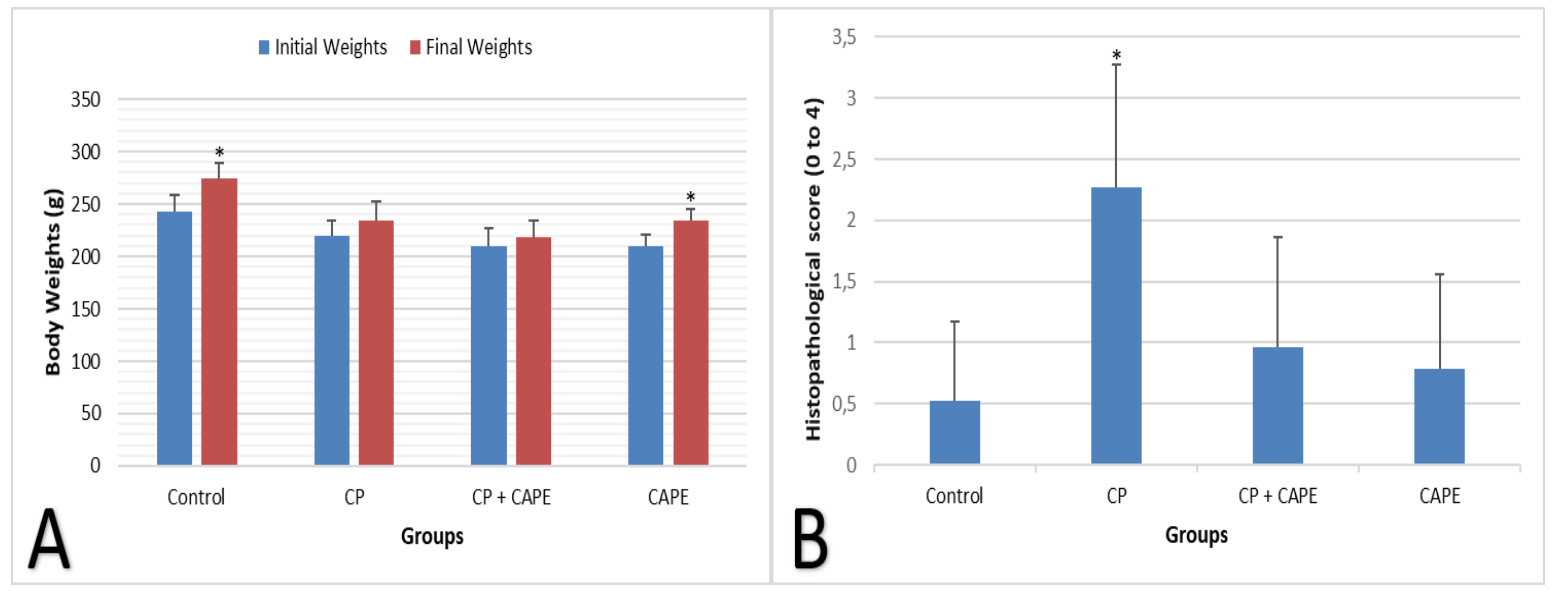

Figure 1: A: Changes in body weight of experimental animals before and after the experimental procedure. B: Liver histopathological scoring. Abbreviations: CP, Cisplatin; CAPE: Caffeic Acid Phenethyl Ester.

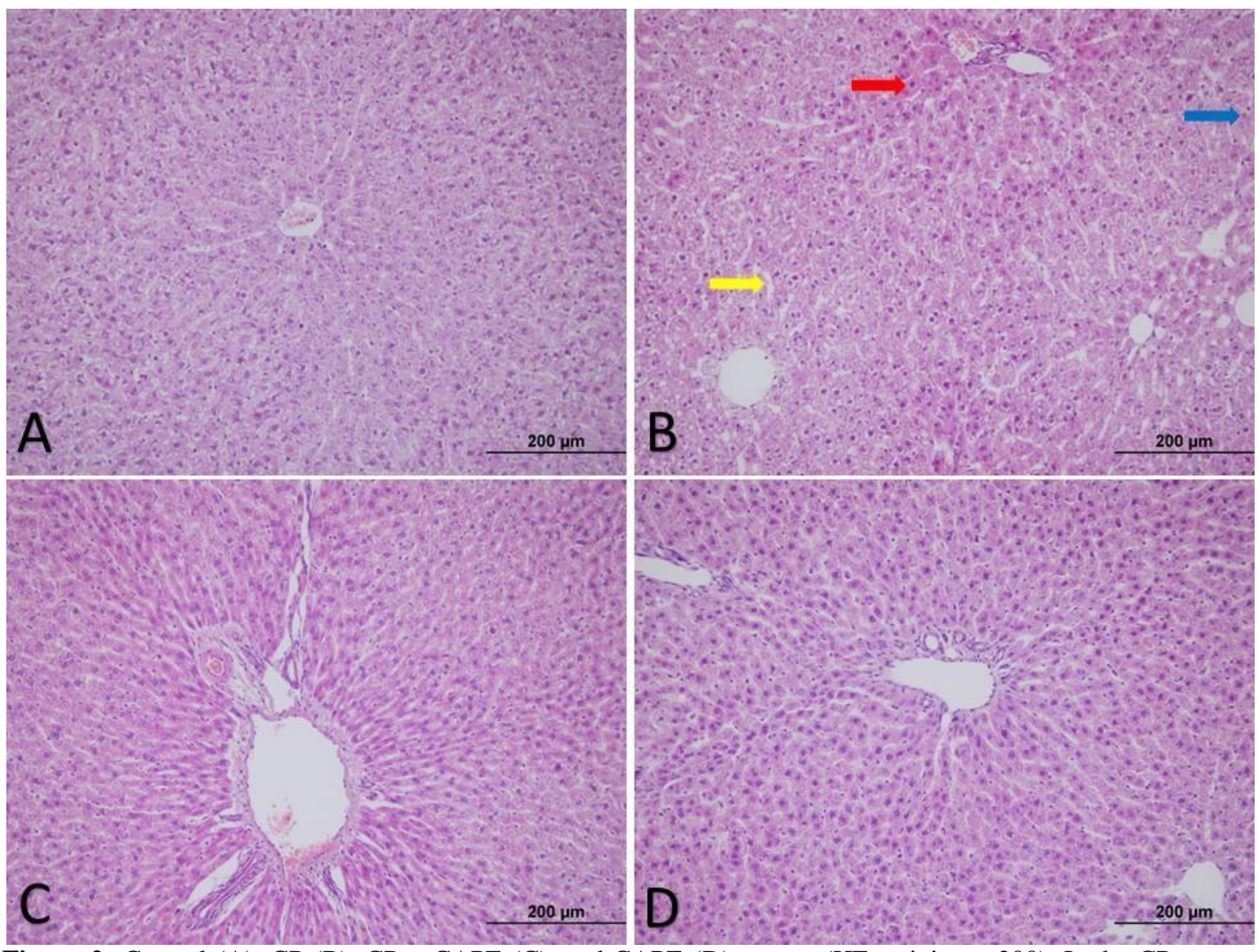

Figure 2: Control (A), CP (B), CP + CAPE (C), and CAPE (D) groups (HE staining, $\times 200)$. In the CP group, hemorrhage (blue arrow), eosinophilic cells (red arrow) and sinusoidal dilatation (yellow arrow) are shown. Abbreviations: CP, Cisplatin; CAPE: Caffeic Acid Phenethyl Ester. 

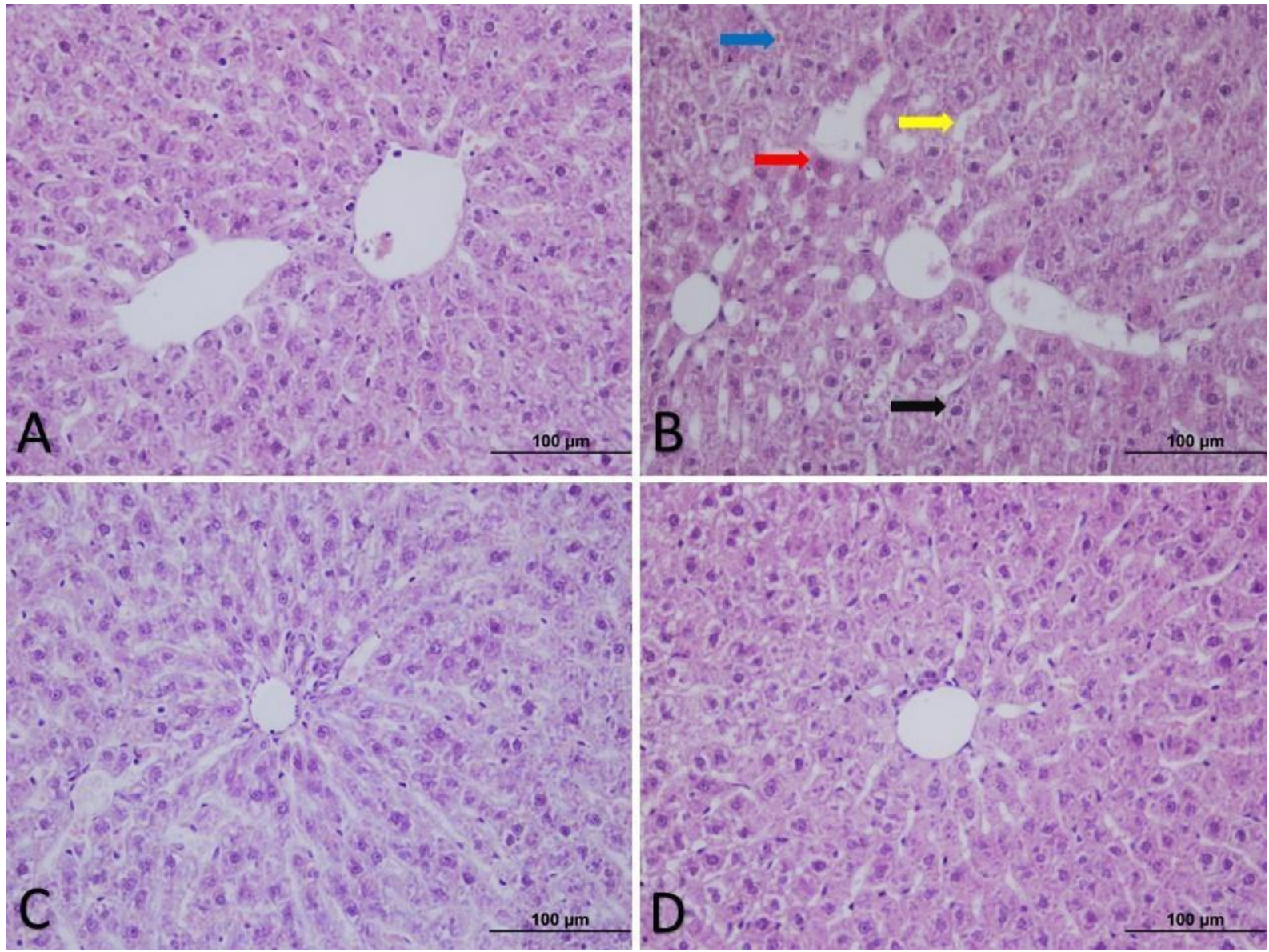

Figure 3: Control (A), CP (B), CP+CAPE (C), and CAPE (D) groups (HE staining, $\times 400$ ). In the CP group, hemorrhage (blue arrow), eosinophilic cells (red arrow), sinusoidal dilatation (yellow arrow) and vacuolization (black arrow) are shown. Abbreviations: CP, Cisplatin; CAPE: Caffeic Acid Phenethyl Ester.

\section{Evaluation of rat body weights}

Body weights of the experimental animals were measured and recorded at the beginning and end of the experiment. Body weights of the animals in the Control and CAPE groups at the end of the experiment increased significantly compared to those on the first day of the experiment $(p<0.001)$. Body weight of the animals both in the $\mathrm{CP}$ group and the $\mathrm{CP}+\mathrm{CAPE}$ group at the end of the experiment increased when compared to those on the first day of the experiment. However, this increase was not statistically significant. The data of animal weights in the experimental groups are shown in Table 1 and Figure 1.

\section{Discussion}

Cancer is one of the most important health problems in the world and is the second leading cause of death in the United States. Cancer is defined as the rapid growth of normal cells in any part of the body out of control. These out-of-control cells can form a mass of tissue called a tumor. Depending on the type of cancer, patients are treated with methods such as radiotherapy, surgery, immunotherapy and chemotherapy (13). CP, cisplatinum or cisdiamminedichloroplatinum (II), is a wellknown chemotherapeutic drug. It is used in the treatment of many types of cancer such as bladder, head and neck, lung, ovarian 
and testicular cancers $(3,14)$. However, negative side effects of $\mathrm{CP}$ such as nephrotoxicity, ototoxicity, hepatotoxicity and cardiomyopathy limit its clinical use (3-5).

Bilgic et al. reported that the necrotic hepatocytes with pycnotic nuclei and eosinophilic hepatocytes in the liver tissue were observed in the single dose of $\mathrm{CP}$ (7 $\mathrm{mg} / \mathrm{kg}$ ) CP administered animals. He also stated that Kuppfer cells increased in the $\mathrm{CP}$ administered group. Overproduction of reactive oxygen species or disruption of antioxidant mechanisms is effective in the formation of this damage in cells. Because reactive oxygen species produced by normal metabolism are eliminated in the liver by some reactions involving enzymes such as superoxide dismutase, catalase, and GSH. Excessive reactive oxygen species cause inflammation and increase cell death by damaging DNA. (15). Boroja et al reported in their study that $\mathrm{CP}$ (single dose, $7.5 \mathrm{mg} / \mathrm{kg}$ ) developed hepatotoxicity through hepatocyte degeneration, necrosis and lymphocyte infiltration in the liver (16). Similar to the studies in the literature, we applied a single dose of CP (7 mg / kg) to animals in our study. According to our histopathological examinations, CP caused severe histopathological effects in the liver tissues in the $\mathrm{CP}$ administered group. These effects were in the form of hemorrhage, sinusoidal dilatation and irregular sinusoids. Furthermore, eosinophilic stained necrotic hepatocytes and vacuolization were observed in addition to other histopathological effects. $\mathrm{CP}$ also affected the increase in weight of the animals fed under normal conditions. The fact that the animal weights in the $\mathrm{CP}$ group did not increase significantly during the experiment indicates that the drug also affected the development of the experimental animals. The negative side effects of $\mathrm{CP}$, which is widely used in cancer patients, on the liver tissue and the disruption of the increase in animal weight limit its use. Elimination of the side effects caused by $\mathrm{CP}$ with various agents will increase the preference of using the drug in the treatment of the disease.

Interesting pharmacological activities from other phenolic components of propolis have been reported in recent studies (17). For this purpose, we investigated the effectiveness of CAPE, one of the active ingredients of propolis, whose effectiveness in many tissues and organs has been investigated. CAPE is one of the active ingredients of propolis and has been used as a folk remedy for many years. CAPE has been shown to inhibit the growth of different types of transformed cells (7). The mechanism of these biological effects of CAPE is not fully known $(8,9)$.

Ferreira et al. applied CAPE at doses of 1, $5,10,25$ and $50 \mu \mathrm{mol}$ against $\mathrm{CP}$-induced neurotoxicity in cell culture models and reported that cell viability increased with a dose of $10 \mu \mathrm{mol}$ (19). Yigit et al. compared the protective effects of low-dose doxycycline and CAPE, reported that CAPE was more protective than doxycycline at low doses. They attributed this effect to the anti-inflammatory, antioxidant and anti-apoptotic properties of CAPE (20). Salmas et al. reported that oxidative changes in kidney tissue of chronic hypertensive rats can be prevented by CAPE administration (21). In our previous study, we observed that CAPE (10 $\mu \mathrm{mol} /$ day) was significantly protective against testicular damage caused by CP (22). According to the literature, CAPE has been shown to have therapeutic effect on many tissues. In our current 
study, we investigated the protective effects of CAPE, one of the active ingredients of propolis, against liver damage caused by $\mathrm{CP}$. According to our results, hemorrhage, sinusoidal dilatation, eosinophilic hepatocytes and vacuolization were reduced in tissue sections belonging to the $\mathrm{CP}+\mathrm{CAPE}$ group. Even in many tissue sections, these harmful effects have disappeared completely. In addition, sinusoids displayed a more regular appearance. Histopathological scoring of the liver was also significantly decreased in the CP + CAPE group compared to the $\mathrm{CP}$ group. This shows that there is a significant reduction in hemorrhage, sinusoidal dilatation, eosinophilic hepatocytes, vacuolization and irregular sinusoids in the tissue. Although CAPE had a corrective effect on the liver, it did not work as an effective agent in increasing animal weight. Long-term effects on metabolism should be investigated in the further studies.

As a result, it was determined that $\mathrm{CP}$ also caused serious histopathological damage in the liver. In this study, it has been shown that CAPE can be protective and corrective against the liver damage caused by CP. Considering the ease of obtaining CAPE and its effectiveness in the clinic, researching the underlying mechanisms will contribute to the literature and public health.

\section{Conflict of interest statement}

The authors declare that they have no conflict of interest.

\section{Acknowledgments}

This study was supported by the Erciyes University Scientific Research Projects Coordination Unit under project TYL2015-5948.

\section{References}

1. Pronk L, Schellens J, Planting AT, et al. Phase I and pharmacologic study of docetaxel and cisplatin in patients with advanced solid tumors. Journal of clinical oncology. 1997;15(3):1071-9.

2. Kelland L. The resurgence of platinumbased cancer chemotherapy. Nature Reviews Cancer. 2007;7(8):573-84.

3. Al-Majed AA. Carnitine deficiency provokes cisplatin-induced hepatotoxicity in rats. Basic \& clinical pharmacology \& toxicology. 2007;100(3):145-50.

4. Amin A, Hamza AA, Kambal A, et al. Herbal extracts counteract cisplatin-mediated cell death in rat testis. Asian journal of andrology. 2008;10(2):291-7.

5. İșeri S, Ercan F, Gedik N, et al. Simvastatin attenuates cisplatin-induced kidney and liver damage in rats. Toxicology. 2007;230(2-3):256-64.

6. Bentli R, Parlakpinar H, Polat A, et al. Molsidomine prevents cisplatin-induced hepatotoxicity. Archives of medical research. 2013;44(7):521-8.

7. Özen S, Akyol O, Iraz M, et al. Role of caffeic acid phenethyl ester, an active component of propolis, against cisplatin-induced nephrotoxicity in rats. Journal of applied toxicology : JAT. 2004;24(1):27-35.

8. Russo A, Longo R, Vanella A. Antioxidant activity of propolis: role of caffeic acid phenethyl ester and galangin. Fitoterapia. 2002;73 Suppl 1:S21-9.

9. Omene C, Kalac M, Wu J, et al. Propolis and its active component, caffeic acid phenethyl ester (CAPE), modulate breast cancer therapeutic targets via an epigenetically mediated mechanism of action. Journal of cancer science \& therapy. 2013;5(10):334.

10. Ogeturk M, Kus I, Colakoglu N, et al. Caffeic acid phenethyl ester protects kidneys against carbon tetrachloride toxicity in rats. Journal of ethnopharmacology. 2005;97(2):273-80.

11. Ceylan T, Ünal MA. Patoloji ve Histoloji Laboratuvarı Uygulama Kitabı. Kapadokya Üniversitesi Yayınları; 2020.

12. Akin AT, Kaymak E, Öztürk E, et al. Investigation of the Therapeutic Effects of Chloroquine in Adriamycin- Induced Hepatotoxicity. The EuroBiotech Journal. 2021;5(1):8-14.

13. Ghosh S. Cisplatin: The first metal based anticancer drug. Bioorganic chemistry. 2019;88:102925.

14. 14. Dasari S, Tchounwou PB. Cisplatin in cancer therapy: molecular mechanisms of action. European journal of pharmacology. 2014;740:364- 78. 
15. Bilgic $\mathrm{Y}$, Akbulut $\mathrm{S}$, Aksungur $\mathrm{Z}$, et al. Protective effect of dexpanthenol against cisplatin-induced hepatotoxicity. Experimental and therapeutic medicine. 2018;16(5):4049-57.

16. Boroja T, Katanić J, Rosić G, et al. Summer savory (Satureja hortensis L.) extract: Phytochemical profile and modulation of cisplatininduced liver, renal and testicular toxicity. Food and ChemicalToxicology. 2018;118:252-63.

17. Russo A, Longo R, Vanella A. Antioxidant activity of propolis: role of caffeic acid phenethyl ester and galangin. Fitoterapia. 2002;73:S21-S9.

18. Ferreira RS, Dos Santos NAG, Martins $\mathrm{NM}$, et al. Caffeic acid phenethyl ester (CAPE) protects PC12 cells from cisplatin-induced neurotoxicity by activating the NGF-signaling pathway. Neurotoxicity Research. 2018;34(1):3246.
19. Yiğit U, Kırzıoğlu FY, Uğuz AC, et al. Is caffeic acid phenethyl ester more protective than doxycycline in experimental periodontitis? Archives of oralbiology. 2017;81:61-8.

20. Salmas RE, Gulhan MF, Durdagi S, et al. Effects of propolis, caffeic acid phenethyl ester, and pollen on renal injury in hypertensive rat: an experimental and theoretical approach. Cell biochemistry and function. 2017;35(6):304-14.

21. Ceylan T, Kaymak E, Tan FC, et al. Research on the protective effect of caffeic acid phenethyl ester on testicular damage caused by cisplatin. Turkish Journal of Medical Sciences. 2020;50(8):2032-9. 